\title{
Complete genome sequence of Halorhabdus utahensis type strain $\left(\mathbf{A X}-2^{\mathrm{T}}\right)$
}

Iain Anderson"1, Brian J. Tindall², Helga Pomrenke², Markus Göker², Alla Lapidus ${ }^{1}$, Matt Nolan ${ }^{1}$, Alex Copeland ${ }^{1}$, Tijana Glavina Del Rio ${ }^{1}$, Feng Chen ${ }^{1}$, Hope Tice ${ }^{1}$, Jan-Fang Cheng ${ }^{1}$, Susan Lucas $^{1}$, Olga Chertkov ${ }^{1,3}$, David Bruce ${ }^{1,3}$, Thomas Brettin ${ }^{1,3}$, John C. Detter ${ }^{1,3}$, Cliff Han $^{1,3}$, Lynne Goodwin ${ }^{1,3}$, Miriam Land ${ }^{1,4}$, Loren Hauser ${ }^{1,4}$, Yun-Juan Chang ${ }^{1,4}$, Cynthia D. Jeffries $^{1,4}$, Sam Pitluck ${ }^{1}$, Amrita Pati ${ }^{1}$, Konstantinos Mavromatis ${ }^{1}$, Natalia Ivanova ${ }^{1}$, Galina Ovchinnikova', Amy Chen ${ }^{5}$, Krishna Palaniappan ${ }^{5}$, Patrick Chain ${ }^{1,6}$, Manfred Rohde ${ }^{7}$ Jim Bristow $^{1}$, Jonathan A. Eisen ${ }^{1,8}$, Victor Markowitz ${ }^{5}$, Philip Hugenholtz ${ }^{1}$, Nikos C. Kyrpides ${ }^{1}$, and Hans-Peter Klenk ${ }^{2 *}$

${ }^{1}$ DOE Joint Genome Institute, Walnut Creek, California, USA

${ }^{2}$ DSMZ - German Collection of Microorganisms and Cell Cultures GmbH, Braunschweig, Germany

${ }^{3}$ Los Alamos National Laboratory, Bioscience Division, Los Alamos, New Mexico, USA

${ }^{4}$ Oak Ridge National Laboratory, Oak Ridge, Tennessee, USA

${ }^{5}$ Biological Data Management and Technology Center, Lawrence Berkeley National Laboratory, Berkeley, California, USA

${ }^{6}$ Lawrence Livermore National Laboratory, Livermore, California, USA

${ }^{7} \mathrm{HZI}$ - Helmholtz Centre for Infection Research, Braunschweig, Germany

${ }^{8}$ University of California Davis Genome Center, Davis, California, USA

*Corresponding author: Hans-Peter Klenk

Keywords: halophile, free-living, non-pathogenic, aerobic, euryarchaeon, Halobacteriaceae

Halorhabdus utahensis Wainø et al. 2000 is the type species of the genus, which is of phylogenetic interest because of its location on one of the deepest branches within the very extensive euryarchaeal family Halobacteriaceae. H. utahensis is a free-living, motile, rod shaped to pleomorphic, Gram-negative archaeon, which was originally isolated from a sediment sample collected from the southern arm of Great Salt Lake, Utah, USA. When grown on appropriate media, $H$. utahensis can form polyhydroxybutyrate (PHB). Here we describe the features of this organism, together with the complete genome sequence, and annotation. This is the first complete genome sequence of the a member of halobacterial genus Halorhabdus, and the 3,116,795 bp long single replicon genome with its 3027 protein-coding and 48 RNA genes is part of the Genomic Encyclopedia of Bacteria and Archaea project.

\section{Introduction}

Strain AX-2 ${ }^{\mathrm{T}}$ (= DSM $12940=$ JCM 11049) is the type strain of the species Halorhabdus utahensis, and represents one of only two species currently assigned to the genus Halorhabdus [1]. Strain AX$2^{\mathrm{T}}$ was first described by Wainø et al. in 2000 [1] as Gram-negative, motile and extremely pleomorphic organism. The organism is of interest because of its position in the tree of life, where the genera Halorhabdus and Halomicrobium constitute one of the deepest branches within the large euryarchaeal family Halobacteriaceae. Here we present a summary classification and a set of features for $H$. utahensis strain $\mathrm{AX}-2^{\mathrm{T}}$ together with the description of the complete genomic sequencing and annotation.

\section{Classification and features}

Only one other $16 \mathrm{~S}$ rRNA encoding sequence has been deposited in the INSDC databases with a similarity of greater than $97 \%$ to that of strain AX$2^{\mathrm{T}}$. That sequence belongs to the other species classified in the genus Halorhabdus, H. tiamatea, which was isolated from a sample of the brinesediment interface of the Shaban Deep in the 
northern Red Sea [2]. With 95\% sequence identity, strain T4.2 (AJ270232), a halophilic archaeon that is neither validly published nor preserved in any collection [3] is the next cultivated neighbor of $H$. utahensis strain $\mathrm{AX}-2^{\mathrm{T}}$. Screening of environmental genomic samples and surveys reported at the NCBI BLAST server indicated no closely related phylotypes ( $>91 \%$ sequence similarity) can be linked to the species or genus.

Figure 1 shows the phylogenetic neighborhood of H. utahensis strain AX-2T in a $16 \mathrm{~S}$ rRNA based tree. The sequence of the unique 16S rRNA gene is identical with the previously published $16 \mathrm{~S}$ rRNA sequence generated from DSM 12940 (AF071880).

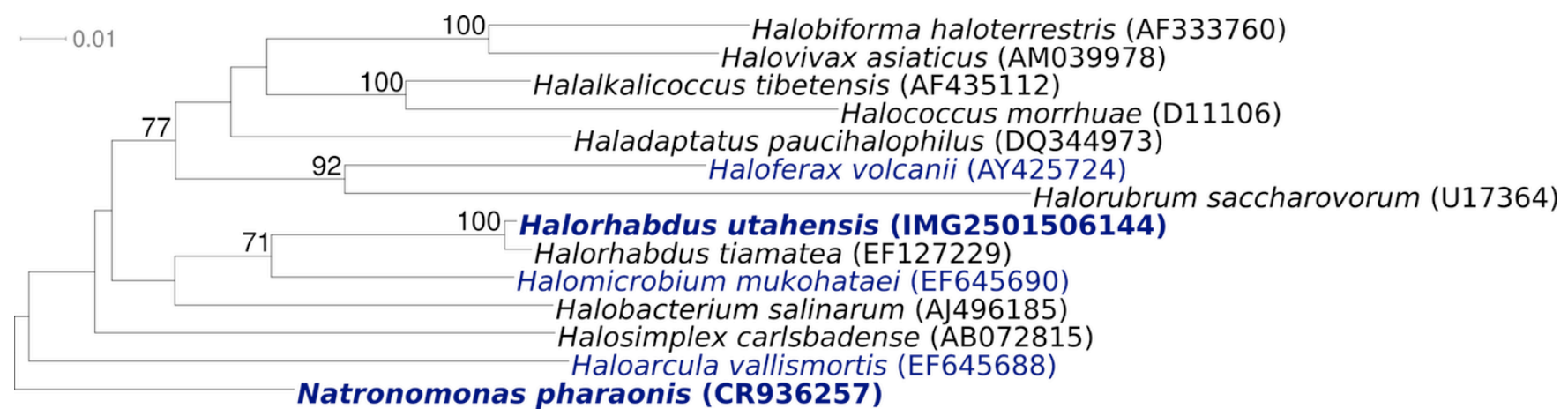

Figure 1. Phylogenetic tree highlighting the position of $H$. utahensis strain $A X-2^{\top}$ with a selection of type strains of the family Halobacteriaceae, inferred from 1,433 aligned $16 \mathrm{~S}$ rRNA characters [4,5] under the maximum likelihood criterion [6]. The tree was rooted with Natronomonas pharaoensis, the deepest branching member of the family Halobacteriaceae. The branches are scaled in terms of the expected number of substitutions per site. Numbers above branches are support values from 1,000 bootstrap replicates, if larger than $60 \%$. Lineages with type strain genome sequencing projects registered in GOLD [7] are shown in blue, published genomes in bold, e.g. the GEBA organism Halomicrobium mukohataei [8].

H. utahensis strain $\mathrm{AX}-2^{\mathrm{T}}$ is rod shaped, but may also form pleomorphic cells (Table 1 and Figure 2). Cells are motile by a single flagellum. Strain AX-2T does not require amino acids for growth and will grow on defined medium containing a nitrogen source, using a single carbon source. Cells may grow anaerobically on glucose by fermentation. Polyhydoxybutyrate inclusions are formed on appropriate media. Spores or other resting stages are not produced. Oxidase and catalase are positive. Cells lyse in distilled water. Gelatin and starch were not hydrolyzed. Proteases not produced and urea was not hydrolyzed; aesculin is hydrolyzed. Esterase, lipase and glucosidase are produced. Arginine dihydrolase is not produced, and consequently arginine does not support anaerobic growth. Ornithine and lysine are not decarboxylated. Growth on glucose, xylose and fructose. Nitrate is reduced to nitrite, but does not support growth [1].

\section{Chemotaxonomy}

Menaquinones are the sole respiratory lipoquinones of $H$. utahensis strain AX-2T. Both MK-8 and MK-8 (VIII- $\mathrm{H}_{2}$ ) are present. The lipids are based on diphytanyl ether lipids. The major phospholipids are the $\mathrm{C}_{20}$ diphytanyl ether analogues of phosphatidylglycerol and methylphosphatidylglycerophosphate (typical of all members of the family Halobacteriaceae), the diether analogue of phosphatidylglycerol sulphate is absent [1]. Two glycolipids have been reported with $R_{f}$ values consistent with their identification as a triglycosyl diphytanyl ether and the sulfated derivative, sulfated triglycosyl diphytanyl. The structures of these two lipids have not been elucidated [1]. The pigments responsible for the red color of the cells have not been recorded, but it may be predicted that they are carotenoids, probably bacterioruberins. Outer cell layers are probably proteinaceous. The presence of peptidoglycan has not been investigated, but is generally absent from members of this family Halobacteriaceae. 


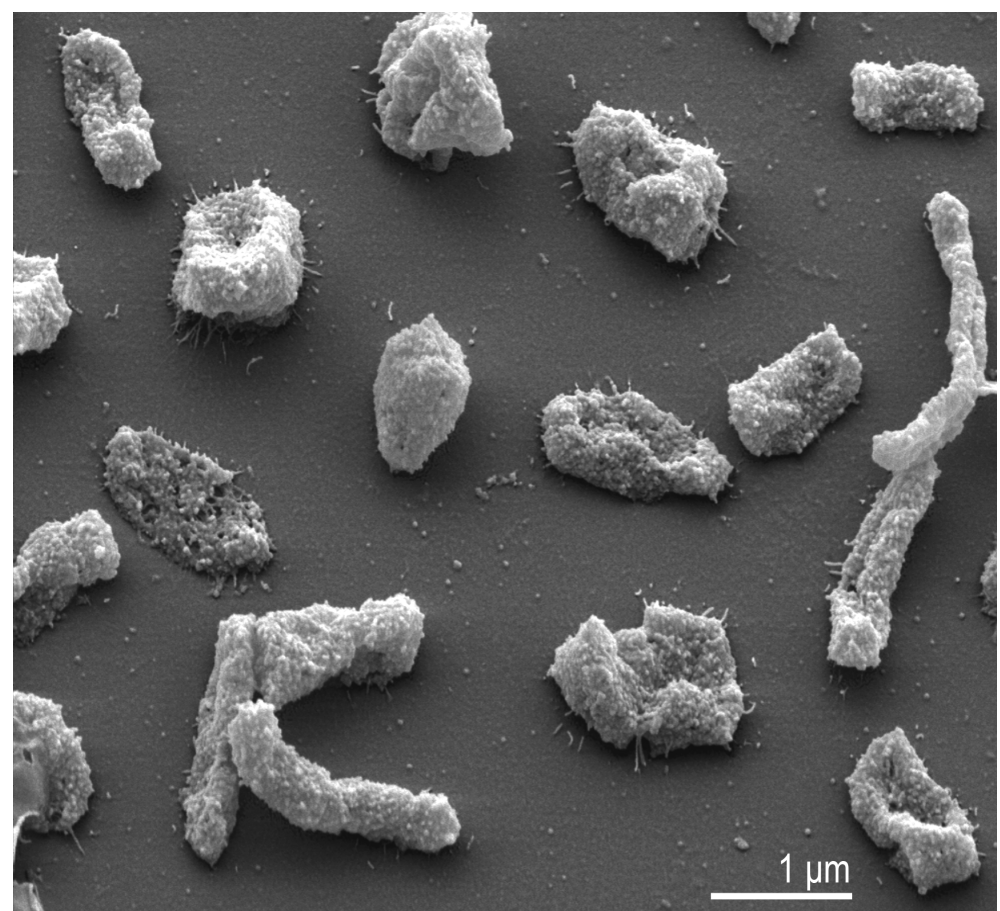

Figure 2. Scanning electron micrograph of $H$. utahensis strain $A X-2^{\top}$

Table 1. Classification and general features of $H$. utahensis strain $A X-2^{\top}$ according to the MIGS recommendations [9]

\begin{tabular}{|c|c|c|c|}
\hline MIGS ID & Property & Term & Evidence code \\
\hline & \multirow{8}{*}{ Classification } & Domain Archaea & TAS [10] \\
\hline & & Phylum Euryarchaeota & TAS $[11,12]$ \\
\hline & & Class Halobacteria & TAS [13] \\
\hline & & Order Halobacteriales & TAS [14] \\
\hline & & Family Halobacteriaceae & TAS [15 \\
\hline & & Genus Halorhabdus & TAS [1] \\
\hline & & Species Halorhabdus utahensis & TAS [1] \\
\hline & & Type strain AX-2 & TAS [1] \\
\hline & Gram stain & negative & TAS [1] \\
\hline & Cell shape & rod to pleomorphic & TAS [1] \\
\hline & Motility & motile by a single flagellum & TAS [1] \\
\hline & Sporulation & nonsporulaing & TAS [1] \\
\hline & Temperature range & mesophile, $15-55^{\circ} \mathrm{C}$ & TAS [1] \\
\hline & Optimum temperature & $50^{\circ} \mathrm{C}$ & TAS [1] \\
\hline \multirow{4}{*}{ MIGS-22 } & Salinity & $\begin{array}{l}\text { halophile, at least } 9 \%(\mathrm{w} / \mathrm{v}) \mathrm{NaCl} \\
\text { maximum } 30 \% \text {, with an optimum at } \\
27 \%\end{array}$ & TAS [1] \\
\hline & Oxygen requirement & $\begin{array}{l}\text { primarily aerobe; facultatively } \\
\text { anaerobic, growth via glucose } \\
\text { fermentation }\end{array}$ & TAS [1] \\
\hline & Carbon source & glucose, xylose and fructose & TAS [1] \\
\hline & Energy source & carbohydrates & NAS \\
\hline MIGS-6 & Habitat & aquatic & TAS [1] \\
\hline MIGS-15 & Biotic relationship & free living & NAS \\
\hline MIGS-14 & Pathogenicity & none & NAS \\
\hline
\end{tabular}


Table 1. Classification and general features of $H$. utahensis strain $A X-2^{\top}$ according to the MIGS recommendations [9] (cont.)

\begin{tabular}{llll}
\hline MIGS ID & Property & Term & Evidence code \\
\hline & Biosafety level & 1 & TAS [16] \\
& Isolation & sediment of Great Salt Lake, Utah & TAS [1] \\
MIGS-4 & Geographic location & sediment of Great Salt Lake, Utah & TAS [1] \\
MIGS-5 & Sample collection time & before 2000 & TAS [1] \\
MIGS-4.1 & Latitude, Longitude & $41.177,-112.502$ & NAS \\
MIGS-4.2 & Depth & sea level & TAS [1] \\
MIGS-4.3 & Altitude & not reported & \\
MIGS-4.4 & &
\end{tabular}

Evidence codes - IDA: Inferred from Direct Assay (first time in publication); TAS: Traceable Author Statement (i.e., a direct report exists in the literature); NAS: Non-traceable Author Statement (i.e., not directly observed for the living, isolated sample, but based on a generally accepted property for the species, or anecdotal evidence). These evidence codes are from the Gene Ontology project [17]. If the evidence code is IDA, then the property was directly observed for a living isolate by one of the authors or an expert mentioned in the acknowledgements.

\section{Genome sequencing and annotation Genome project history}

This organism was selected for sequencing on the basis of each phylogenetic position, and is part of the Genomic Encyclopedia of Bacteria and Archaea project. The genome project is deposited in the Genome OnLine Database [7] and the complete genome sequence in GenBank. Sequencing, finishing and annotation were performed by the DOE Joint Genome Institute (JGI). A summary of the project information is shown in Table 2.

Table 2. Genome sequencing project information

\begin{tabular}{|c|c|c|}
\hline MIGS ID & Property & Term \\
\hline MIGS-31 & Finishing quality & Finished \\
\hline MIGS-28 & Libraries used & $\begin{array}{l}\text { Three genomic libraries: two Sanger libraries ( } 8 \mathrm{~kb} \\
\text { pMCL200 and fosmid pcc1Fos) and one } 454 \\
\text { pyrosequence standard library }\end{array}$ \\
\hline MIGS-29 & Sequencing platforms & ABI3730, 454 GS FLX \\
\hline MIGS-31.2 & Sequencing coverage & 8.3x Sanger; $21.2 \times$ pyrosequence \\
\hline MIGS-30 & Assemblers & Newbler version 1.1.02.15, phrap \\
\hline \multirow[t]{6}{*}{ MIGS-32 } & Gene calling method & Prodigal, GenePRIMP \\
\hline & INSDC ID & СР001687 \\
\hline & Genbank Date of Release & August 27, 2009 \\
\hline & GOLD ID & Gc01053 \\
\hline & NCBI project ID & 29305 \\
\hline & Database: IMG-GEBA & 2501416929 \\
\hline \multirow[t]{2}{*}{ MIGS-13 } & Source material identifier & DSM 12940 \\
\hline & Project relevance & Tree of Life, GEBA \\
\hline
\end{tabular}

\section{Growth conditions and DNA isolation}

H. utahensis strain AX-2 ${ }^{\mathrm{T}}$, DSM 12940, was grown in DSMZ medium 927 (H. utahensis medium) [18] at $40^{\circ} \mathrm{C}$. DNA was isolated from $1-1.5 \mathrm{~g}$ of cell paste using a Qiagen Genomic 500 DNA Kit
(Qiagen, Hilden, Germany) as described in Wu et al. [19].

\section{Genome sequencing and assembly}

The genome was sequenced using a combination of Sanger and 454 sequencing platforms. All general aspects of library construction and 
sequencing performed at the JGI can be found on the JGI website (http://www.jgi.doe.gov/). 454 Pyrosequencing reads were assembled using the Newbler assembler, version 1.1.02.15 (Roche). Large Newbler contigs were broken into 3,474 overlapping fragments of $1,000 \mathrm{bp}$ and entered into assembly as pseudo-reads. The sequences were assigned quality scores based on Newbler consensus q-scores with modifications to account for overlap redundancy and to adjust inflated qscores. A hybrid 454/Sanger assembly was made using the parallel phrap assembler (High Performance Software, LLC). Possible misassemblies were corrected with Dupfinisher or transposon bombing of bridging clones [20]. Gaps between contigs were closed by editing in Consed, custom primer walk or PCR amplification. A total of 212 Sanger finishing reads were produced to close gaps, to resolve repetitive regions, and to raise the quality of the finished sequence. The final assembly consists of 26,545 Sanger and 382,722 pyrosequence (454) reads. Together all sequence types provided $29.5 \times$ coverage of the genome. The error rate of the completed genome sequence is less than 1 in 100,000.

\section{Genome annotation}

Genes were identified using Prodigal [21] as part of the Oak Ridge National Laboratory genome annotation pipeline, followed by a round of manual curation using the JGI GenePRIMP pipeline (http://geneprimp.jgi-psf.org/) [22]. The predicted CDSs were translated and used to search the National Center for Biotechnology Information (NCBI) nonredundant database, UniProt, TIGRFam, Pfam, PRIAM, KEGG, COG, and InterPro databases. Additional gene prediction analysis and functional annotation was performed within the Integrated Microbial Genomes Expert Review platform (http://img.jgi.doe.gov/er) [23].

\section{Genome properties}

The genome is $3,116,795$ bp long and comprises one main circular chromosome with a $62.9 \%$ GC content (Table 3 and Figure 3). Of the 3,075 genes predicted, 3,027 were protein coding genes, and 48 RNAs; 29 pseudogenes were also identified. The majority of the protein-coding genes $(60.5 \%)$ were assigned with a putative function, while those remaining were annotated as hypothetical proteins. The distribution of genes into COGs functional categories is presented in Table 4.

Table 3.

\begin{tabular}{lrr}
\hline Attribute & Value & \% of Total \\
\hline Genome size (bp) & $3,116,795$ & $100.00 \%$ \\
DNA Coding region (bp) & $2,768,833$ & $88.83 \%$ \\
DNA G+C content (bp) & $1,960,463$ & $62.90 \%$ \\
Number of replicons & 1 & \\
Extrachromosomal elements & 0 & \\
Total genes & 3,075 & $100.00 \%$ \\
RNA genes & 48 & $1.59 \%$ \\
rRNA operons & 1 & \\
Protein-coding genes & 3,027 & $98.30 \%$ \\
Pseudo genes & 29 & $1.90 \%$ \\
Genes with function prediction & 1,860 & $60.47 \%$ \\
Genes in paralog clusters & 473 & $15.38 \%$ \\
Genes assigned to COGs & 1,946 & $63.28 \%$ \\
Genes assigned Pfam domains & 1,918 & $62.37 \%$ \\
Genes with signal peptides & 705 & $22.93 \%$ \\
Genes with transmembrane helices & 782 & $25.43 \%$ \\
CRISPR repeats & 2 & \\
\hline
\end{tabular}




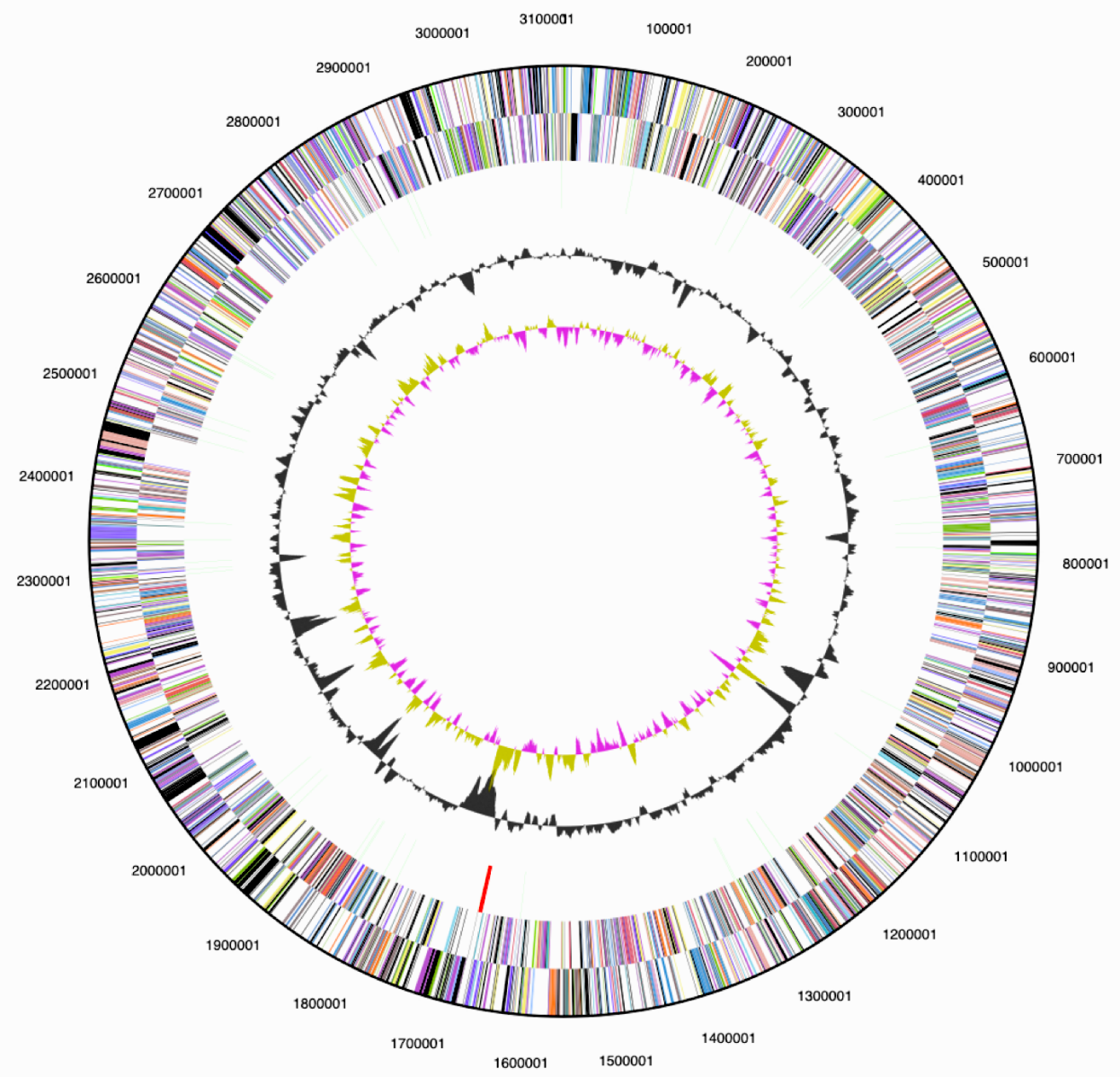

Figure 3. Graphical circular map of the genome. From outside to the center: Genes on forward strand (color by COG categories), Genes on reverse strand (color by COG categories), RNA genes (tRNAs green, rRNAs red, other RNAs black), GC content, GC skew.

Table 4. Number of genes associated with the general COG functional categories

\begin{tabular}{lrrl}
\hline Code & Value & \% age & Description \\
\hline J & 157 & 5.2 & Translation, ribosomal structure and biogenesis \\
A & 1 & 0.0 & RNA processing and modification \\
K & 120 & 3.9 & Transcription \\
L & 115 & 3.8 & Replication, recombination and repair \\
B & 3 & 0.0 & Chromatin structure and dynamics \\
D & 26 & 0.8 & Cell cycle control, mitosis and meiosis \\
Y & 0 & 0.0 & Nuclear structure \\
V & 41 & 1.3 & Defense mechanisms \\
T & 121 & 4.0 & Signal transduction mechanisms \\
M & 82 & 2.7 & Cell wall/membrane biogenesis \\
N & 33 & 1.0 & Cell motility \\
Z & 0 & 0.0 & Cytoskeleton \\
W & 0 & 0.0 & Extracellular structures \\
U & 25 & 0.8 & Intracellular trafficking and secretion \\
O & 95 & 3.1 & Posttranslational modification, protein turnover, chaperones \\
C & 147 & 4.8 & Energy production and conversion \\
\hline
\end{tabular}


Table 4. Number of genes associated with the general COG functional categories

\begin{tabular}{lrrl}
\hline Code & Value & \% age & Description \\
\hline G & 107 & 3.5 & Carbohydrate transport and metabolism \\
E & 165 & 5.4 & Amino acid transport and metabolism \\
F & 65 & 2.1 & Nucleotide transport and metabolism \\
H & 106 & 3.5 & Coenzyme transport and metabolism \\
I & 42 & 1.4 & Lipid transport and metabolism \\
P & 122 & 4.0 & Inorganic ion transport and metabolism \\
Q & 24 & 0.8 & Secondary metabolites biosynthesis, transport and catabolism \\
R & 0 & 10.9 & General function prediction only \\
S & 214 & 7.1 & Function unknown \\
- & 1,081 & 35.7 & Not in COGs \\
\hline
\end{tabular}

\section{Acknowledgements}

We gratefully acknowledge the help of Susanne Schneider (DSMZ) for DNA extraction and quality analysis. This work was performed under the auspices of the US Department of Energy's Office of Science, Biological and Environmental Research Program, and by the University of California, Lawrence Berkeley

\section{References}

1. Wainø M, Tindall BJ, Ingvorsen K. Halorhabdus utahensis gen. nov., sp. nov., an aerobic, extremely halophilic member of the Archaea from Great Salt Lake, Utah. Int J Syst Evol Microbiol 2000; 50:183190. PubMed

2. Antunes A, Taborda M, Huber R, Moissl C, Nobre MF, da Costa MS. Halorhabdus tiamatea sp. nov., a non-pigmented, extremely halophilic archaeon from a deep-sea, hypersaline anoxic basin of the Red Sea, and emended description of the genus Halorhabdus. Int J Syst Evol Microbiol 2008; 58:215-220. PubMed doi:10.1099/ijs.0.65316-0

3. McGenity TJ, Gemmell RT, Grant WD, Stan-Lotter H. Origins of halophilic microorganisms in ancient salt deposits. Environ Microbiol 2000; 2:243-250. PubMed doi:10.1046/j.1462-2920.2000.00105.x

4. Lee C, Grasso C, Sharlow MF. Multiple sequence alignment using partial order graphs. Bioinformatics 2002; 18:452-464. PubMed doi:10.1093/bioinformatics/18.3.452

5. Castresana J. Selection of conserved blocks from multiple alignments for their use in phylogenetic analysis. Mol Biol Evol 2000; 17:540-552. PubMed

6. Stamatakis A, Hoover P, Rougemont J. A rapid bootstrap algorithm for the RAxML web-servers. Syst Biol 2008; 57:758-771. PubMed doi:10.1080/10635150802429642
National Laboratory under contract No. DE-AC0205CH11231, Lawrence Livermore National Laboratory under Contract No. DE-AC52-07NA27344, and Los Alamos National Laboratory under contract No. DEAC02-06NA25396, as well as German Research Foundation (DFG) INST 599/1-1.

7. Liolios K, Mavromatis K, Tavernarakis N, Kyrpides NC. The Genomes OnLine Database (GOLD) in 2007: status of genomic and metagenomic projects and their associated metadata. Nucleic Acids Res 2008; 36:D475-D479. PubMed doi:10.1093/nar/gkm884

8. Tindall BJ, Schneider S, Lapidus A, Copeland A, Glavina Del Rio T, Nolan M, Lucas S Cheng F, Saunders E, Bruce D., et al. Complete genome sequence of Halomicrobium mukohataei type strain (arg-2T). Stand Genomic Sci 2009; 1:

9. Field D, Garrity G, Gray T, Morrison N, Selengut J, Sterk P, Tatusova T, Thomson N, Allen MJ, Angiuoli SV, et al. Towards a richer description of our complete collection of genomes and metagenomes: the "Minimum Information about a Genome Sequence" (MIGS) specification. Nat Biotechnol 2008; 26:541-547. PubMed doi:10.1038/nbt1360

10. Woese CR, Kandler O, Wheelis ML. Towards a natural system of organisms: proposal for the domains Archaea, Bacteria, and Eucarya. Proc Natl Acad Sci USA 1990; 87:4576-4579. PubMed doi:10.1073/pnas.87.12.4576

11. Garrity GM, Holt JG. Phylum All. Euryarchaeota phy. nov. In Bergey's Manual of Systematic Bacteriology, vol. 1. 2nd ed. Edited by: Garrity, GM, Boone, DR and Castenholz, RW. Springer, New York; 2001:211-355. 
12. List Editor. Validation of publication of new names and new combinations previously effectively published outside the IJSEM. Validation List no. 85. Int J Syst Evol Microbiol 2002; 52:685-690. PubMed doi:10.1099/ijs.0.02358-0

13. Garrity GM, Lilburn TG, Cole JR, Harrison SH, Euzeby J, Tindall BJ. "Part 1- The Archaea, Phyla Crenarchaeota and Euryarchaeota" Taxonomic Outline of the Bacteria and Archaea. 2007. www.taxonomicoutline.org

14. Grant WD, Larsen H. Group III. Extremely halophilic archaeobacteria. Order Halobacteriales ord. nov. In: JT Staley, MP Bryant, N Pfennig \& JG Holt (eds) Bergey's Manual of Systematic Bacteriology, first edition, Vol 3, The Williams \& Watkins Co., Baltimore, 1989, pp 2216-2228.

15. Gibbons NE. Family V. Halobacteriaceae fam. nov. In: RE Buchanan \& NE Gibbons (eds) Bergey's Manual of Determinative Bacteriology, eighth edition, The Williams \& Watkins Co., Baltimore, 1974, pp 279.

16. Anonymous. Biological Agents: Technical rules for biological agents. www.baua.de TRBA 466.

17. Ashburner M, Ball CA, Blake JA, Botstein D, Butler $\mathrm{H}$, Cherry JM, Davis AP, Dolinski K, Dwight SS, Eppig JT, et al. Gene ontology: tool for the unification of biology. Nat Genet 2000; 25:25-29. PubMed doi:10.1038/75556
18. List of growth media used at DSMZ: http://www.dsmz.de/microorganisms/media_list.php

19. Wu M, Hugenholtz P, Mavromatis K, Pukall R, Dalin E, Ivanova N, Kunin V, Goodwin L, Wu M, Tindall BJ, et al. A phylogeny-driven genomic encyclopedia of Bacteria and Archaea. Nature (In press).

20. Sims D, Brettin T, Detter JC, Han C, Lapidus A, Copeland A, Glavina Del Rio T, Nolan M, Chen F, Lucas $\mathrm{S}$, et al. Complete genome of Kytococcus sedentarius type strain $\left(541^{\mathrm{T}}\right)$.. Stand Genomic Sci 2009; 1:12-20. doi:10.4056/sigs.761

21. Anonymous. Prodigal Prokaryotic Dynamic Programming Genefinding Algorithm. Oak Ridge National Laboratory and University of Tennessee 2009 http://compbio.ornl.gov/prodigal/

22. Pati A, Ivanova N, Mikhailova N, Ovchinikova G, Hooper SD, Lykidis A, Kyrpides NC. GenePRIMP: A Gene Prediction Improvement Pipeline for microbial genomes. 2009 (Submitted)

23. Markowitz VM, Mavromatis K, Ivanova NN, Chen IMA, Chu K, Kyrpides NC. Expert Review of Functional Annotations for Microbial Genomes. Bioinformatics 2009; 25:2271-2278. PubMed doi:10.1093/bioinformatics/btp393 